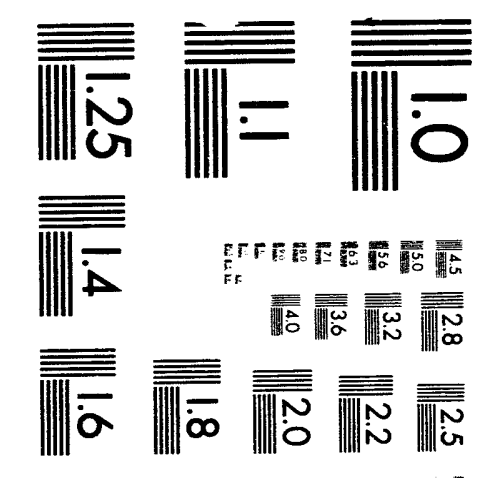



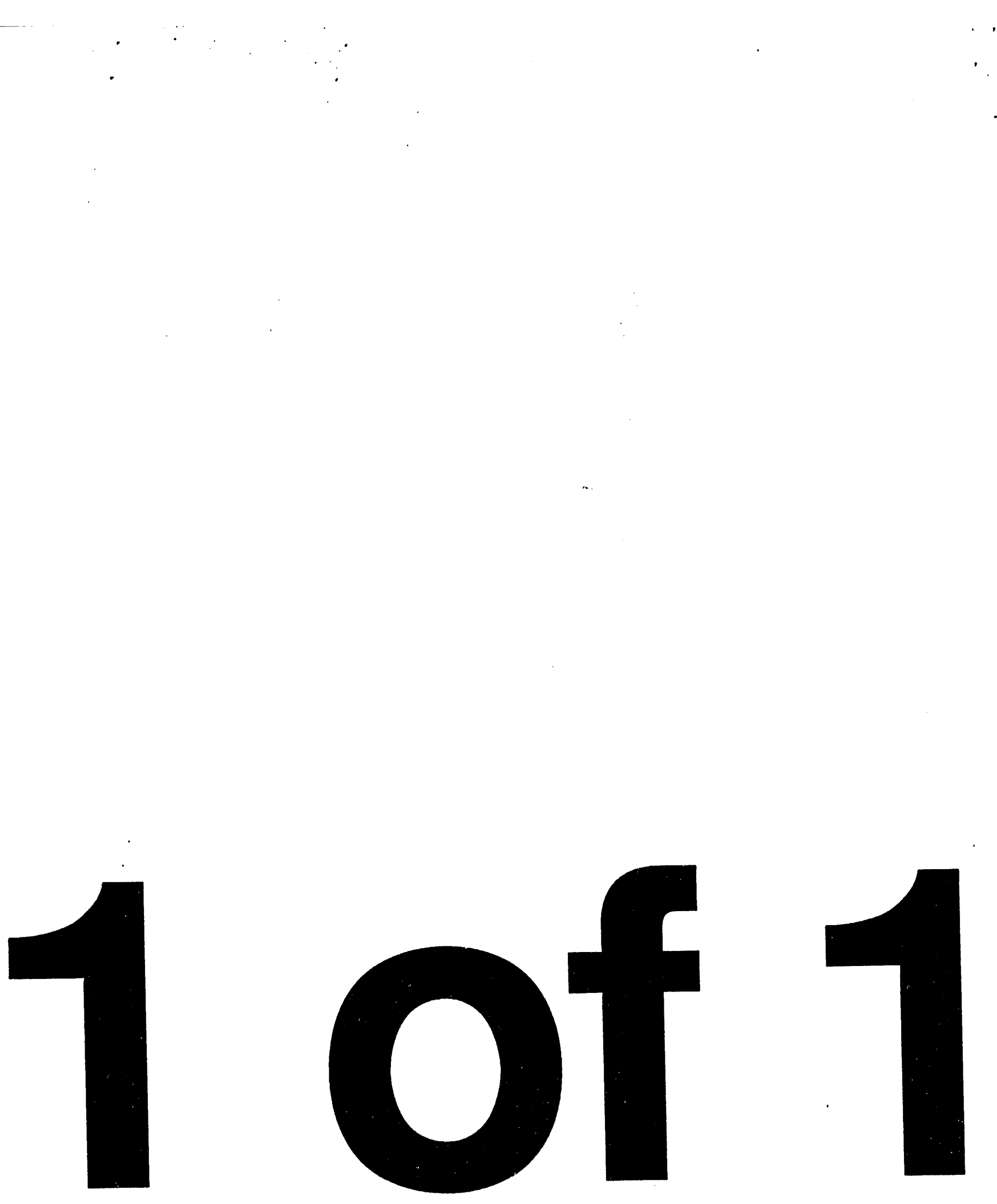

. 
LBL 34017

\title{
CRYOGENIC FRACTURE BEHAVIOR OF 316LN IN MAGNETIC FIELDS UP TO 14.6T
}

\author{
J.W. Chan, D. Chu, C. Tseng and J.W. Morris, Jr.
}
Department of Materials Science and Mineral Engineering
University of California
and

Center for Advanced Materials

Materials Sciences Division

Lawrence Berkeley Laboratory

University of California

Berkeley, CA 94720

July 1993

This work was supported by the Director, Office of Energy Research, Office of Fusion Energy, Development and Technology Divison of the U.S. Department of Energy under Contract No. DE-AC03-76F00098

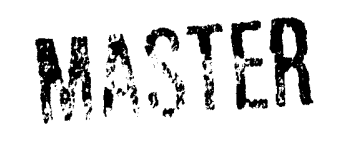




\title{
CRYOGENIC FRACTURE BEHAVIOR OF 316LN IN MAGNETIC FIELDS UP TO14.6 T
}

\author{
J. W. Chan, D. Chu, C. Tseng, and J. W. Morris, Jr. \\ Department of Materials Science and Mineral Engineering \\ University of California, Berkeley \\ and \\ Center for Advanced Materials \\ Lawrence Berkeley Laboratory
}

\begin{abstract}
Some of the proposed magnet case alloys for the International Thermonuclear Experimental Reactor (ITER) are metastable austenitic stainless steels. The case will be subjected to high loads and can be as much as $100 \mathrm{~mm}$ thick. Case assembly will probably require thick section welding with its attendant chemical inhomogeneity. Even alloys that are thermally stable with respect to martensitic transformation can, under these conditions of high stresses, cryogenic temperature, and high magnetic fields, undergo transformation. Previous work at $8 \mathrm{~T}$ has shown a measurable magnetic field effect on the $4.2 \mathrm{~K}$ tensile and fracture toughness properties of AISI300 series metastable austenitic stainless steels. Thus, it is important to understand how these alloys will behave under conditions of high magnetic fields and cryogenic temperature, particularly at the higher magnetic fields that will be used in ITER. $4.2 \mathrm{~K}$ data for $316 \mathrm{LN}$ indicates that the change in fracture toughness is not monotonic. It initially decreases and then later increases with applied field.
\end{abstract}

\section{INTRODUCTION}

Austenitic stainless steels have been used as structural alloys in high field superconducting magnets. A modified AISI316LN, a metastable austenitic stainless steel, is a candidate structural alloy for ITER. ${ }^{1}$ In this application the alloy will be 
required to sustain high stresses in an environment of high magnetic fields at $4.2 \mathrm{~K}$. Plastic deformation at low temperatures induces martensitic transformation in these metastable alloys. The presence of a strong magnetic field enhances this transformation, since the formation of a ferromagnetic phase such as $\alpha^{\prime}$ martensite in the magnetic field reduces the overall free energy. A phase transformation during service may result in unexpected changes in mechanical properties such as tensile, fracture, or fatigue, that may degrade the performance of the device. Even if the base alloys are used in stress-temperature-magnetic field conditions that do not induce a phase transformation, any vields that are in the structure remain suspect since solidification induced segregation can produce local chemical inhomogeneity that can change the stability locally. It is thus necessary to characterize the mechanical behavior of these alloys at the anticipated operating conditions and to better understand the mechanisms responsible for the behavior. Prior work indicates that the presence of a strong magnetic field can influence the cryogenic fracture 2,3 and tensile ${ }^{4-6}$ behavior of AISI304-type stainless steels in a strong magnetic field. The change in the $4.2 \mathrm{~K}$ mechanical properties is due to an enhanced martensitic transformation during deformation. Both increases 3,7 and decreases 2 in $4.2 \mathrm{~K}$ fracture toughness were observed in an $8 \mathrm{~T}$ magnetic field, relative to $0 \mathrm{~T}$, with the direction of fracture toughness change being influenced both by the stability of the alloys and by the specimen preparation conditions, such as fatigue precracking temperature. Some of the mechanisms affecting the fracture toughness changes that arise because of the in situ phase transformation were identified in previous work ${ }^{3}$. Here, the $4.2 \mathrm{~K}$ fracture toughness of AISI316LN in magnetic fields of up to $14.6 \mathrm{~T}$ are examined.

\section{EXPERIMENTAL}

The composition of the $316 \mathrm{LN}$ plate used in this work is listed in Table 1. It was

Table 1. Alloy composition in wt.\%, balance Fe.

\begin{tabular}{cccccccccccccc}
\hline $\mathrm{Cr}$ & $\mathrm{Ni}$ & $\mathrm{Mo}$ & $\mathrm{Mn}$ & $\mathrm{Si}$ & $\mathrm{Cu}$ & $\mathrm{V}$ & $\mathrm{Al}$ & $\mathrm{Ti}$ & $\mathrm{N}$ & $\mathrm{C}$ & $\mathrm{P}$ & $\mathrm{O}$ & $\mathrm{S}$ \\
\hline 17.15 & 12.88 & 2.45 & 1.45 & 0.32 & 0.17 & 0.07 & 0.04 & 0.01 & 0.166 & 0.023 & 0.030 & 0.002 & $<.005$ \\
\hline
\end{tabular}

tested in the as-received condition. Specimens were machined from a nominally $30 \mathrm{~mm}$ thick plate. The tensile specimens were in the transverse orientation, and the CT specimens were in the T-L orientation. Both tensile and $\mathrm{J}_{\mathrm{IC}}$ tests were performed on specimens taken from this plate. The tensile tests were performed at $4.2 \mathrm{~K}$ with no applied magnetic field while the $\mathrm{J}_{\mathrm{IC}}$ tests were performed at $4.2 \mathrm{~K}$ with applied magnetic fields of $8,8.4,12.2$, and 14.6 T. The specimens were oriented such that the axis of the solenoidal field was parallel to the specimen load direction. Two series of tests were performed with this plate. The first set used CT specimens with nominal 
dimensions of $50 \mathrm{~mm} \times 54 \mathrm{~mm} \times 24 \mathrm{~mm}$. Because of the magnet cold bore size considerations, the second set used slightly smaller CT specimens with nominal dimensions of $43 \mathrm{~mm} \times 45 \mathrm{~mm} \times 14 \mathrm{~mm}$. The first set was tested in a $100 \mathrm{~mm}$ diameter working bore, $9 \mathrm{~T}$ superconducting magnet while the second set was tested in a $78 \mathrm{~mm}$ diameter working bore, $15 \mathrm{~T}$ Bitter magnet. The thickness and ligament dimensions met the criteria required by ASTM E813-88 for the strength and toughness levels measured. The tensile specimens were $6.4 \mathrm{~mm}$ diameter round specimens with a $25.4 \mathrm{~mm}$ gage length. The stroke rate for both tests was $2.5 \times 10^{-3} \mathrm{~mm} / \mathrm{sec}$. The CT specimens were precracked at room temperature to a nominal $\mathrm{a} / \mathrm{w}$ of 0.6 .

The effects of the magnetic field on the clip-on strain gages were accounted for by calibrating the gages at $4.2 \mathrm{~K}$ in the various magnetic fields by means of a micrometer with a long extension. The response of the gages remained linear, although the output per unit displacement changed with the strength of the magnetic field.

In the first set of tests, the effect of the fringe magnetic field on the load cell was determined by loading a dummy aluminum specimen in the grips to a known constant stroke value and then moving the entire load train from its raised position above the magnet to its operating position and observing for changes in load. No load changes were observable during the traverse, indicating no effect. The LVDT that was in the position control loop had been checked for fringe field effects by comparing its output at known displacements both in and away from the load train's operating positions. No observable effects were evident.

In the second set of tests, the fringe field effects were checked by placing a hydraulic piston in series with the load train. A pressure transducer was connected to the piston through a $9 \mathrm{~m}$ long hydraulic hose and was thus well away from any appreciable fringe fields. The outputs from the load cell and piston were then compared, both with and without a magnetic field. The ratio of the load cell output to the pressure transducer output remained constant for all magnet currents and loads used, indicating negligible fringe field effects.

\section{RESULTS}

The $4.2 \mathrm{~K}$ tensile yield stress for this plate is $1030 \mathrm{MPa}$, and the tensile stress is $1590 \mathrm{MPa}$ with a uniform elongation of $65 \%$, total elongation of $75 \%$, and a reduction in area of $49 \%$. The fracture toughness results from both sets of tests are shown in Figure 1. The shaded symbols represent the data from the first set of tests on larger CT specimens while the open symbols are data from the second set of tests. The error bars indicate the spread in the data where more than one valid test result is available.

The data show a nonmonotonic change in fracture toughness with applied magnetic field. It shows an apparent decrease from $0 \mathrm{~T}$ to about $8 \mathrm{~T}$ followed by an increase in the fracture toughness to magnetic fields up to $14.6 \mathrm{~T}$. The fracture surface morphologies reflect the differences. 


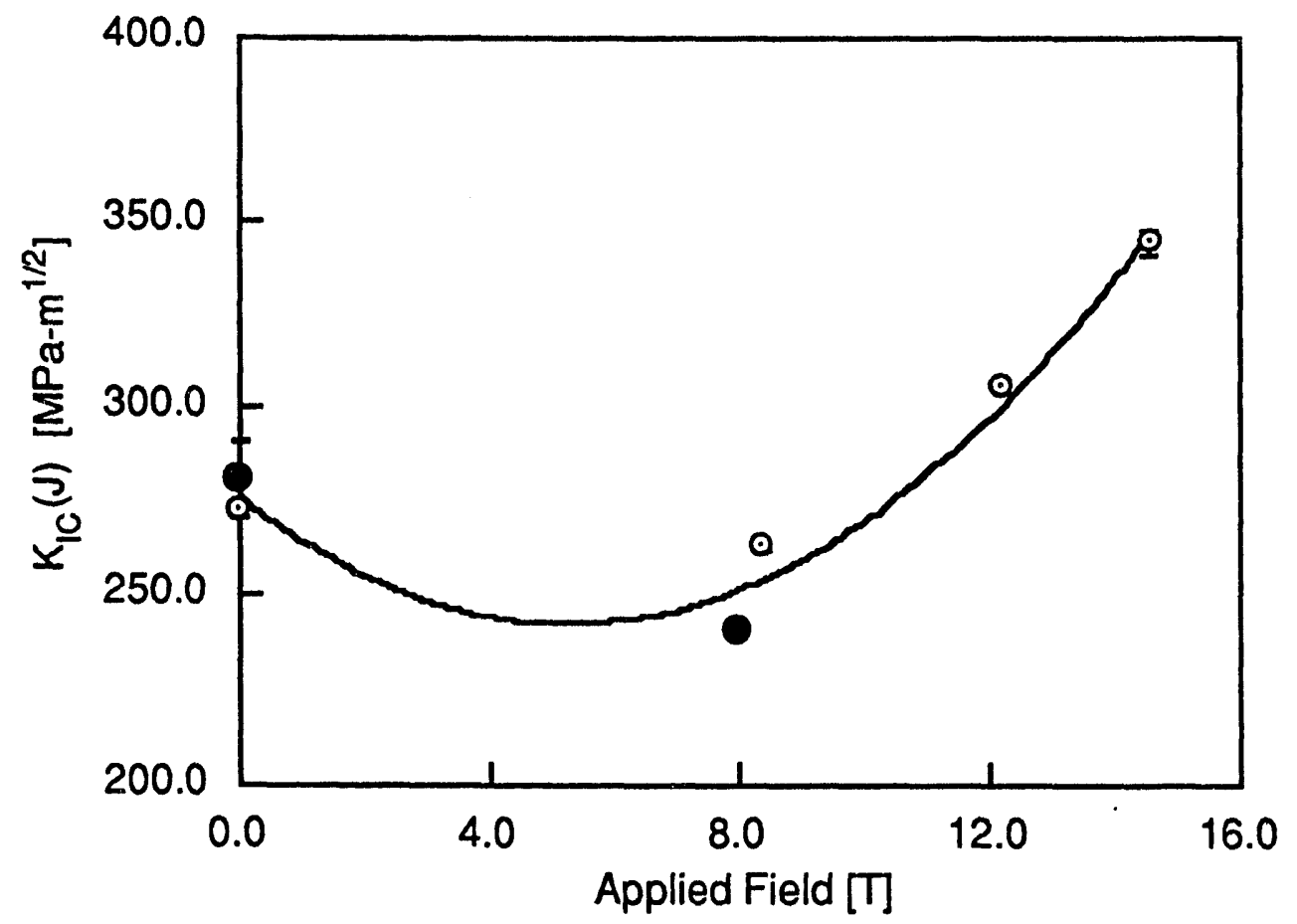

Figure 1. $4.2 \mathrm{~K}$ fracture toughness, $\mathrm{K} I \mathrm{IC}(\mathrm{J})$, of $316 \mathrm{LN}$ as a function of applied magnetic field. The closed and the open circles represent data taken from slightly different size CT specimens. The larger samples are represented by the closed circles. The error bars denote the spread in the data where there are more than one samples tested per condition.

Figure 2 compares the fracture surfaces from an $8 \mathrm{~T}$ specimen with that of the higher toughness $14.6 \mathrm{~T}$ specimen. The fracture surface of the $8 \mathrm{~T}$ specimen has more flat-wall dimples and, in general, a more planar appearance than the high toughness specimen. This is consistent with a lower energy crack propagation path. X-ray diffraction results from the fracture surfaces show the presence of $\alpha^{\prime}$ martensite on the fracture surfaces of all cases.

\section{DISCUSSTON}

Prior work on 304 and 304L stainless steels has shown that the $4.2 \mathrm{~K}$ fracture toughness is dependent on the strength of the applied field during deformation. Most of the members of the AISI 300 series of stainless steel are metastable and undergo a phase transition from an fcc structure to a more stable bec martensite on deformation at low temperatures. The transformation is driven by the free energy difference between the bcc $\alpha^{\prime}$ phase and the fcc $\gamma$ phase, and opposed by the increase in strain and surface energies produced by the transformation. The presence of an applied magnetic field during deformation will increase the driving force for the phase transformation since a ferromagnetic $\alpha^{\prime}$ phase will be produced from the $\gamma$ phase, and the alignment of the 
magnetic moments with the magnetic field will reduce the free energy of the system. Thus, the applied magnetic field would tend to enhance and alter the timing of the transformation. This change in transformation behavior in the presence of a strong magnetic field was proposed as the cause of the observed fracture toughness change in AISI304 and AISI304L.
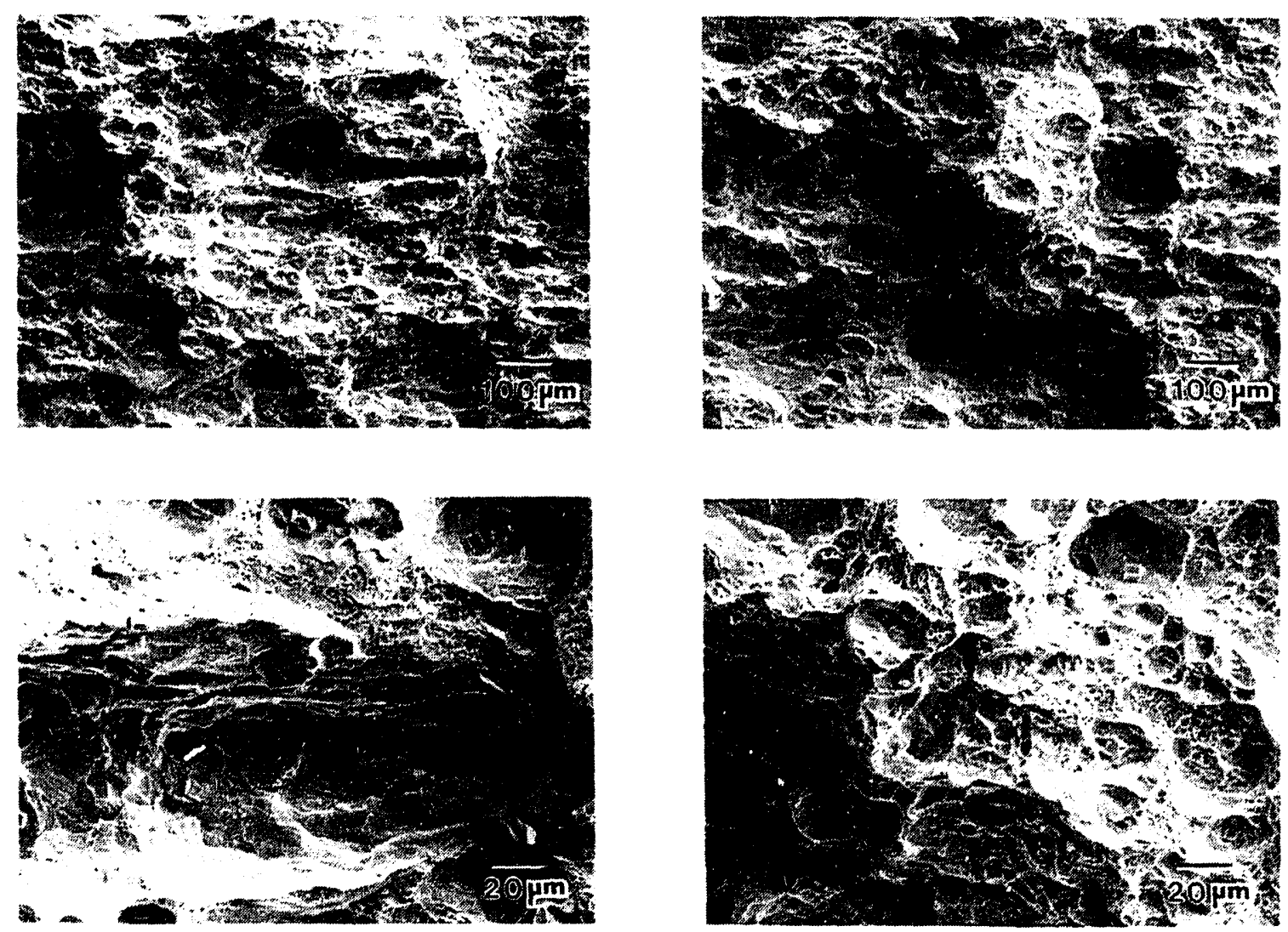

XBB938-5622

Figure 2. SEM fractographs of an area just ahead of the precrack tip at the midplane of the CT specimens. Crack propagation direction is from right to left. The $8 \mathrm{~T}$ fracture surface is on the left and the $14.6 \mathrm{~T}$ surface is on the right. The $8 \mathrm{~T}$ surface has a more planar appearance with more planar microvoids surrounded by more ductile areas.

Figure 1 shows a nonmonotonic change in fracture toughness for AISI316LN with increasing magnetic field. The $\mathrm{K}_{\mathrm{IC}}(\mathrm{J})$ values decrease $10 \%$ from approximately $280 \mathrm{MPa}-\mathrm{m}^{1 / 2}$ to $250 \mathrm{MPa}-\mathrm{m}^{1 / 2}$ on going from $0 \mathrm{~T}$ to $8 \mathrm{~T}$, and then increase $23 \%$ from the $0 \mathrm{~T}$ condition to $345 \mathrm{MPa}-\mathrm{m}^{1 / 2}$ at $14.6 \mathrm{~T}$. The current fracture toughness data on AISI316LN is consistent with the previous observations on AISI304 and AISI304L and with the proposed explanation for the observed behavior. This type of behavior should be expected since there are a number of different factors that can affect the observed fracture toughness. ${ }^{3}$ Two important, opposing, transformation related, concurrently operating, factors affecting the fracture toughness under these test conditions will be 
discussed here. The first is the local stress relaxation due to the transformation strains that serves to increase fracture toughness. The transformation strains that occur within the plastic zone constrained by the surrounding elastic portion of the specimen, when properly timed, will reduce the effective stress on the crack tip, increasing the measured fracture toughness. The contribution of this mechanism to the measured fracture toughness will eventually saturate at high $\alpha^{\prime}$ volume fractions since further increments of $\alpha^{\prime}$ formation would become increasingly more difficult. In addition, the transformation should not necessarily occur simultaneously and uniformly over the entire plastic zone, but probably occurs sequentially over small, noncontiguous, interacting, local volumes within the plastic zone. This interaction will affect the details of the local stress state and strain hardening rate and thus the deformation process within the fracture process zone. The transformation strains can be regarded as an additional localized deformation mechanism, and thus will affect the measured fracture toughness. Evidence for this is seen on the fracture surfaces, where there are relatively planar regions surrounded by more ductile microvoid containing regions. There is an observed difference in the fracture surface morphologies of the $14.6 \mathrm{~T}$ and the $8 \mathrm{~T}$ specimens, the ones with the greatest fracture toughness difference. The $8 \mathrm{~T}$ specimen fracture surfaces contain more and larger regions of relatively planar areas compared to the more ductile microvoid containing areas than the $14.6 \mathrm{~T}$ specimen.

The second transformation related factor important to the fracture behavior is the formation of lower toughness $\alpha^{\prime}$ martensite ahead of the crack tip in the process zone. The contribution of this factor to the fracture toughness is negative if the transformation occurs in a volume far enough ahead of the crack tip such that the tensile stress peak of the crack tip stress field can operate on the transformed volume to cause brittle failure of the transformed region. If the transformation occurs after the tensile stress peak has passed, the formed martensite will not be subjected to the hydrostatic tensile stress peak and may not fail in a brittle manner. However, the decrease in fracture toughness due to this mechanism cannot be linear with applied magnetic field or alloy stability. It has to saturate to some constant value characteristic of the $\alpha^{\prime}$ martensite at very high fields or low stability, i.e. at volume fractions of $\alpha^{\prime}$ close to 1 , since $\alpha^{\prime}$ production will saturate and a further increment in the amount of $\alpha^{\prime}$ will have a negligible contribution to the aggregate contributions of the $\alpha^{\prime}$ areas already present. Similarly, there should also be a plateau in the fracture toughness versus stability curve at the high stability (or low applied field) end, i.e. near $\alpha^{\prime}$ volume fractions of 0 . At small volume fractions the locally transformed volumes, unless they are closely spaced or contiguous, will behave more like widely spaced inclusions, with the properties of the matrix dominating the fracture behavior. Thus, the fracture toughness contribution from this mechanism as a function of increasing applied magnetic field or decreasing stability should be sigmoidal in nature and negative in direction, with the size of the initial plateau a function of the timing and the spacing of the local transformation volumes. The rate of decrease will be a function of the inherent strength of the $\alpha^{\prime}$ martensite and its spatial distribution and rate of formation, and of the stress distribution ahead of the crack.

Ignoring the secondary effects discussed in a prior work ${ }^{3}$, the measured fracture toughness behavior is thus the sum of the two opposite trending effects discussed above. The sum of these effects can yield a fracture toughness versus applied magnetic field curve that can dip, as seen in the $316 \mathrm{LN}$ data, or that can produce a rise at 
intermediate fields, depending on the relative rate of change from the two contributions discussed. This is illustrated schematically in Figure 3. The fracture toughness is expected to saturate to some constant value at higher fields since both contributions will saturate at higher fields as the transformation becomes nearly complete.
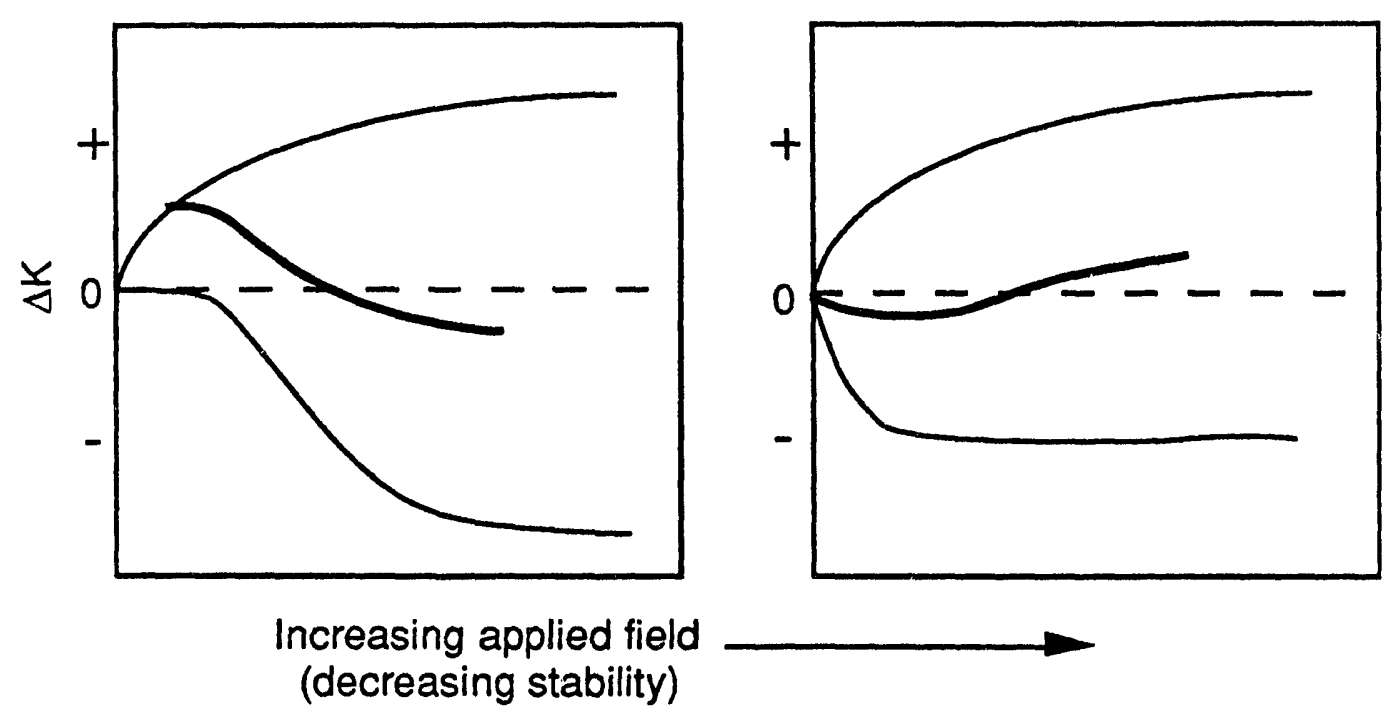

Figure 3. Schematic drawing illustrating the effects the varying relative rates of the development of the two fracture toughness components can have on the fracture toughness behavior with increasing applied magnetic fields. The heavy curve represents the sum of the contributions from the two components.

\section{CONCLUSIONS}

AISI316LN shows a nonmonotonic increase in the measured fracture toughness at $4.2 \mathrm{~K}$ with increasing magnetic fields. The fracture toughness decreases until approximately $8 \mathrm{~T}$ and then rises $23 \%$ above its $0 \mathrm{~T}$ value at $14.6 \mathrm{~T}$. This behavior is a function of the alloy's stability, the timing of the martensite transformation and the inherent fracture toughness level of the $\alpha$ martensite.

\section{ACKNOWLEDGMENTS}

Part of this work was performed at the Francis Bitter National Magnet Laboratory, which is supported at M.I.T. by the National Science Foundation. The alloy used in this work was supplied by Dr. R. Tobler of the National Institute of Standards and Technclogy. This work was supported by the Director, Office of Fusion Research, 
J. W. Chan, D. Chu, C. Tseng, J. W. Morris, Jr., Cryogenic Fracture Behavior of 316LN in Magnetic Fields up to $14.6 \mathrm{~T}$

Office of Fusion Energy, Development and Technology Division, U. S. Department of Energy, under Contract No. DE-AC03-76SF00098.

\section{REFERENCES}

1. L.T. Summers, J. R. Miller, and J. R. Heim, "The International Thermonuclear Experimental Reactor (ITER); Design and Materials Selection," Adv. Cryo. Eng., 36:769 (1990).

2. E., Fukushima, S. Kobatake, M. Tanaka, and H. Ogiwara, "Fractıre Toughness Tests on 304 Stainless Steel in High Magnetic Fields at Cryogenic Temperatures, " Adv. Cryo. Eng., 34:367 (1988).

3. J. W. Chan, J. Glazer, Z. Mei, J. W. Morris, Jr., "4.2 K Fracture Toughness of 304 Stainless Steel in a Magnetic Field, "Adv. Cryo. Eng., 36:1299 (1989).

4. B. Fultz and J. W. Morris, Jr., "Effects of High Magnetic Fields on the Flow Stress of 18-8 Stainliess Steels, "Acta Metall., 34:379 (1986).

5. B. Fultz, G.O. Fior, G. M. Chang, R. Kopa, and J. W. Morris, Jr., "MagnetoMechanical Effects in Two Steels with Metastable Austenite, "Adv. Cryo. Eng., 32:377 (1986).

6. B. Fultz, G. M.Chang, R. Kopa, and J. W. Morris, Jr., "Magneto-Mechanical Effects in 304 Stainless Steels," Adv. Cryo. Eng., 30:253 (1983).

7. E., Fukushima, S. Kobatake, M. Tanaka, and H. Ogiwara, "Fractue Toughness of 304 Stainless Steel in High Magnetic Fields at Cryogenic Temperature, " $11^{\text {th }}$ Conference on Magnet Technology, Tsukuba, Japan, Sept 1989. 

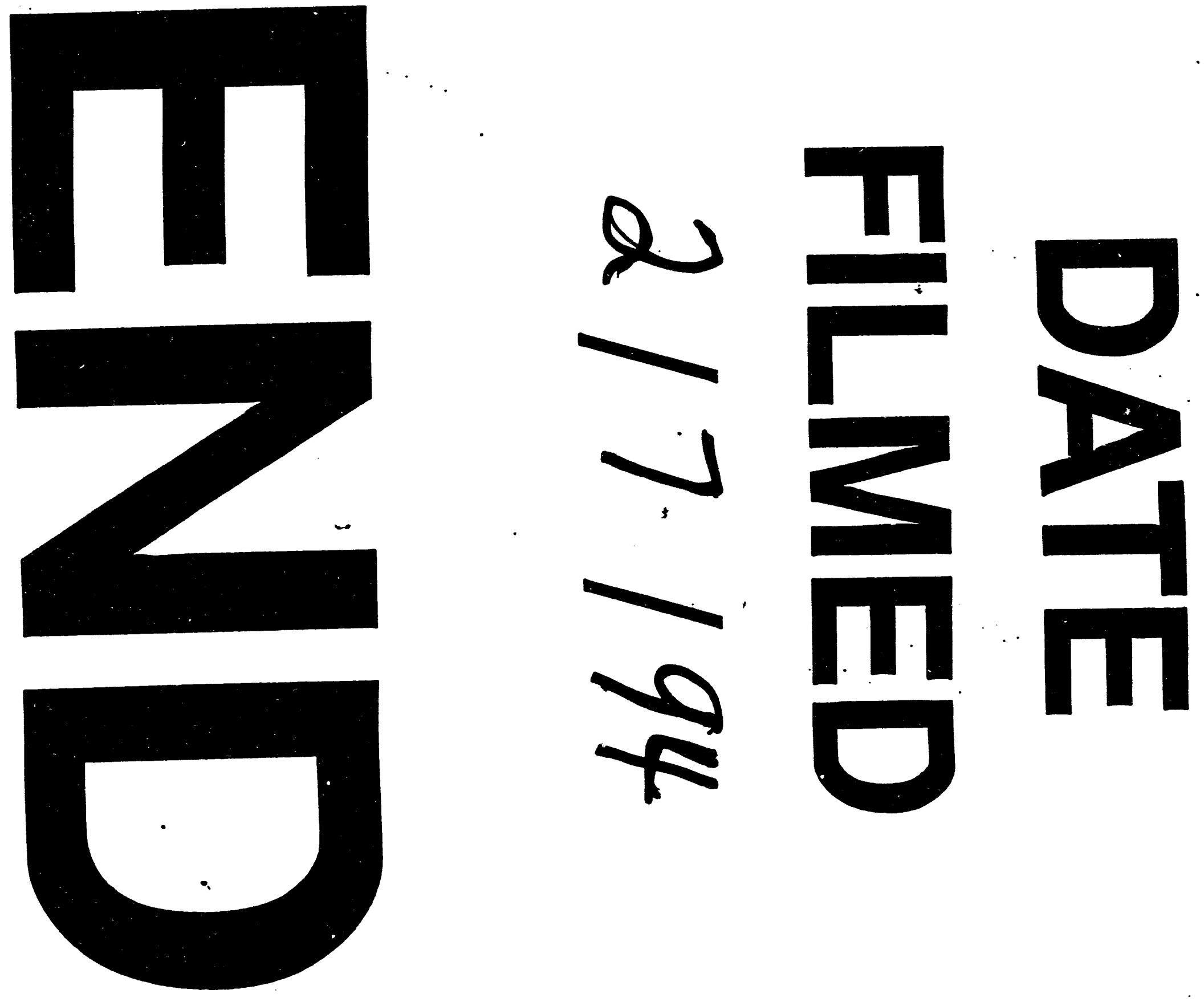


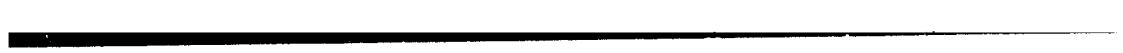

